\title{
Pediatric stroke associated with a sedentary lifestyle during the SARS-CoV-2 (COVID-19) pandemic: a case report on a 17-year-old
}

\author{
Keng Lam ${ }^{1}$ (D) Jonathan H. Lee ${ }^{1} \cdot$ Pamela Cheng $^{1} \cdot$ Zahra Ajani $^{1} \cdot$ Morris M. Salem ${ }^{2} \cdot$ Navdeep Sangha $^{1}$
}

Received: 10 September 2020 / Accepted: 23 October 2020 / Published online: 28 October 2020

(C) Fondazione Società Italiana di Neurologia 2020

\begin{abstract}
The severe acute respiratory syndrome coronavirus 2 (SARS-CoV-2) pandemic-associated quarantine has led to a more sedentary lifestyle in teenagers. This may increase the risk for venous thromboembolism and a subsequent source of an ischemic stroke through a patent foramen ovale (PFO). Here, we report a pediatric stroke case likely due to these factors.
\end{abstract}

Keywords Neurology $\cdot$ Stroke $\cdot$ Patent foramen ovale $\cdot$ COVID $\cdot$ Thromboembolism

\author{
Abbreviations \\ S A R S-Co V-Severe acute respiratory syndrome coronavi- \\ 2 rus 2 \\ COVID-19 Coronavirus disease 2019 \\ PFO Patent foramen ovale \\ NIHSS National Institutes of Health Stroke Scale \\ CT Computed tomography \\ MR Magnetic resonance
}

\section{Introduction}

The severe acute respiratory syndrome coronavirus 2 (SARSCoV-2) pandemic, also known as coronavirus disease 2019 (COVID-19), has led to a higher prevalence of diminished physical activity. A recent study focusing on adults showed that daily step counts have decreased worldwide [1]. However, research on the pediatric population remains lacking. A study on children with congenital heart disease supports decreased Fitbit step counts during the COVID-19 pandemic [2]. Meanwhile, consumer spending on video games in

Keng Lam

keng.lam@kp.org

1 Department of Neurology, Kaiser Permanente, Los Angeles Medical Center, 1505 Edgemont, 5th Floor, Los Angeles, CA 90027, USA

2 Department of Pediatric Cardiology, Kaiser Permanente, Los Angeles Medical Center, Los Angeles, CA, USA the first quarter of 2020 has increased compared to last year [3]. Hence, we suspect that adolescents are overall more immobile as the result of COVID-19 public health quarantine measures. Decreased mobility could be considered a risk factor for venous thromboembolism even for the pediatric population [4], resulting in an ischemic stroke through a patent foramen ovale (PFO) [5]. Here, we report a pediatric stroke case likely due to factors described above.

\section{Case report}

A 17-year-old boy without significant past medical history developed acute onset of blurry vision, difficulty speaking, right facial droop, and right upper and lower extremity weakness and numbness while playing video games. He was immediately taken to a hospital where he was evaluated by a telestroke neurologist and found to have a National Institutes of Health Stroke Scale (NIHSS) score of 16. Computed tomography (CT) of the head showed no intracranial hemorrhage. He received intravenous tissue plasminogen activator. Subsequently, a CT angiogram of the head and neck was performed and showed a left P1 segment filling defect (Fig. 1), but he did not receive thrombectomy. He was then transferred to the regional Comprehensive Stroke Center. Magnetic resonance (MR) imaging of the brain revealed diffusion restriction in the anterior medial left thalamus extending to the thalamocapsular junction. On further interview, parents reported that the patient had been playing video games excessively and sitting in the same spot for $10 \mathrm{~h}$ daily. This was not 


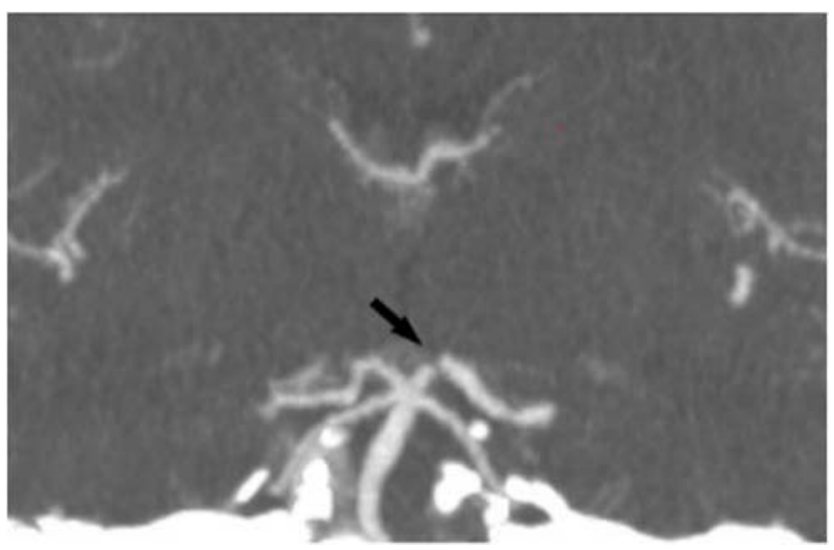

Fig. 1 Filling defect in the left P1 segment

the case pre-pandemic, where he was more active with dancing. He had a body mass index of 18 (weight $57 \mathrm{~kg}$ and height $1.75 \mathrm{~m}$ ). Family reported no history of early vascular disease.

Serum studies were collected to evaluate for hypercoagulability and arterial inflammatory diseases including anticardiolipin antibody, beta 2-glycoprotein, lupus anticoagulant, fasting homocysteine, antithrombin III, protein S, protein $\mathrm{C}$, activated protein $\mathrm{C}$ resistance, factor $\mathrm{V}$ Leiden, prothrombin 20210, factor VIII, d-dimer, antinuclear antibodies, anti-neutrophil cytoplasmic antibody, and erythrocyte sedimentation rate. They were all normal. COVID-19 testing was also negative. Echocardiogram showed a hypermobile atrial septum with a 3.2-mm opening consistent with a PFO. Without Valsalva, there was a mild-to-moderate amount of right-to-left shunt at baseline; with Valsalva, there was a moderate amount of right-to-left shunt. Further imaging with lower extremity Doppler and MR of the pelvis did not reveal any deep vein thrombosis. Repeat vessel imaging with MR angiography of the brain and neck did not reveal stenosis, occlusion, or luminal irregularities; notable was that the previously seen left P1 filling defect was no longer present. There was no arrhythmia detected during his hospital course. Patient's imaging did not support an arterial (i.e., dissection, focal cerebral arteriopathy, atherosclerotic disease, vasculitis) etiology as a cause of the stroke. The resolved left P1 filling defect on subsequent imaging pointed toward an embolic nature of the stroke.

Over the following 2 days, his NIHSS score continues to improve and he had a score of 3 on discharge. He was prescribed daily aspirin $81 \mathrm{mg}$ and instructed to follow-up with outpatient pediatric cardiology for a PFO closure.

\section{Discussion}

Cryptogenic stroke comprises about $25 \%$ of all ischemic strokes in adults, with experts advocating for revising the classification framework of stroke by adding on PFO-associated stroke as a distinct entity [6]. Studies have shown that closure of PFO for adults with cryptogenic ischemic stroke is associated with a lower rate of recurrent infarcts compared to medical therapy alone [7]. The most recent American Academy of Neurology practice guideline recommends practitioners to consider PFO closure for patients younger than age 60 with no other identified mechanism of their embolic-appearing ischemic infarct [8]. There are no controlled studies of PFO closure for children with embolic stroke; hence, optimal treatment is unclear. Nevertheless, most case reports describe closure as the planned treatment [9].

In our case, we suspect the patient suffered an arterial ischemic infarct from a PFO with venous thromboembolism as the source precipitated by decreased mobility during the COVID-19 pandemic. A lack of deep vein thrombosis on our workup does not exclude venous thromboembolism; studies have shown less than $50 \%$ of patients with proven pulmonary embolism have deep vein thrombosis [10], suggesting that a source of embolism is often not found. Among the teenagers, sedentary lifestyle, especially when associated with video gaming, has been identified as a modifiable risk factor for venous thromboembolism and reported in multiple case reports $[11,12]$. Our case demonstrates that the pandemic has had far-reaching effects, possibly leading to an increased stroke risk among the young due to a sedentary lifestyle.

Authors' contributions K.L., J.L., P.C., Z.A., and M.S. evaluated the patient and participated in direct care. K.L. and J.L. wrote and edited the manuscript. P.C. and N.S. revised the manuscript. K.L. prepared and submitted the final version of the manuscript.

\section{Compliance with ethical standards}

Conflict of interest The authors declare that they have no conflict of interest.

Ethics approval/consent Ethics approval and consent not required.

\section{References}

1. Tison G, Avram R, Kuhar P et al (2020) Worldwide effect of COVID-19 on physical activity: a descriptive study. Ann Intern Med. https://doi.org/10.7326/m20-2665

2. Hemphill N, Kuan M, Harris K (2020) Reduced physical activity during COVID-19 pandemic in children with congenital heart disease. Can J Cardiol 36(7):1130-1134. https://doi.org/10.1016/j. cjca.2020.04.038

3. The NPD Group: quarterly U.S. consumer spend on video game products reached the highest total in U.S. history in first quarter of 2020. The NPD Group. https://www.npd.com/wps/portal/npd/us/ news/press-releases/2020/quarterly-us-consumer-spend-on-videogame-products-reached-the $\% 2 \mathrm{D} \% 2 \mathrm{Dhighest}$-total-in-us-historyin-first-quarter-of-2020. Published 2020. Accessed Aug 28, 2020.

4. Mahajerin A, Croteau S (2017) Epidemiology and risk assessment of pediatric venous thromboembolism. Front Pediatr 5. https://doi. org/10.3389/fped.2017.00068 
5. Tsze D, Valente J (2011) Pediatric stroke: a review. Emerg Med Int 2011:1-10. https://doi.org/10.1155/2011/734506

6. Elgendy AY, Saver JL, Amin Z et al (2020) Proposal for updated nomenclature and classification of potential causative mechanism in patent foramen ovale-associated stroke [published online ahead of print, 2020 Apr 13]. JAMA Neurol. https://doi.org/10.1001/ jamaneurol.2020.0458

7. Saver J, Carroll J, Thaler D et al (2017) Long-term outcomes of patent foramen ovale closure or medical therapy after stroke. $\mathrm{N}$ Eng1 J Med 377(11):1022-1032. https://doi.org/10.1056/ nejmoa1610057

8. Messé S, Gronseth G, Kent D et al (2020) Practice advisory update summary: patent foramen ovale and secondary stroke prevention. Neurology. 94(20):876-885. https://doi.org/10.1212/wnl. 0000000000009443
9. Dowling M, Ikemba C (2011) Intracardiac shunting and stroke in children: a systematic review. J Child Neurol 26(1):72-82. https:// doi.org/10.1177/0883073810383913

10. van Rossum A, van Houwelingen H, Kieft G, Pattynama P (1998) Prevalence of deep vein thrombosis in suspected and proven pulmonary embolism: a meta-analysis. Br J Radiol 71(852):12601265. https://doi.org/10.1259/bjr.71.852.10318998

11. Kohorst MA et al (2018) Obesity, sedentary lifestyle, and video games: the new thrombophilia cocktail in adolescents. Pediatr Blood Cancer 65(7):e27041

12. Rambaran KA, Alzghari SK (2020) Gamer's thrombosis: a review of published reports. Ochsner J 20(2):182-186

Publisher's note Springer Nature remains neutral with regard to jurisdictional claims in published maps and institutional affiliations. 